\title{
WHEN IS A NAKAYAMA CLOSURE SEMIPRIME?
}

\author{
JANET C. VASSILEV
}

\begin{abstract}
Many well-known closure operations such as integral closure and tight closure are both semiprime operations and Nakayama closures. In this short note, we begin the study on the overlap between Nakayama closures and semiprime operations. We exhibit examples of closure operations which are either semiprime or Nakayama but not the other. In the case of a discrete valuation ring we show that a closure operation $c$ is Nakayama if and only if it is semiprime and

$$
(0)^{c}=\bigcap_{n \geq 1}\left(I^{n}\right)^{c}
$$
\end{abstract}

for any ideal $I$.

1. Introduction. Closure operations and their related constructs on commutative rings and modules have been linked to classifying singularities, proving some difficult theorems like the Briançon-Skoda theorem and some versions of the homological conjectures. In recent years, interest has been sparked to study closure operations in their own right.

Many well-known closure operations $c$ are semiprime operations, meaning they preserve products, $\left(I^{c} J^{c}\right)^{c}=(I J)^{c}$. In addition, on local rings $(R, \mathfrak{m})$, they are Nakayama, that is, if $I \subseteq J \subseteq(I+\mathfrak{m} J)^{c}$, then $I^{c}=J^{c}$. These properties may not seem at all related, but we would like to determine what conditions force a closure operation to satisfy both properties.

We first look at the case when $(R, \mathfrak{m})$ is a discrete valuation ring and observe that a closure operation $c$ is Nakayama if and only if it is

2010 AMS Mathematics subject classification. Primary 13A15,13C05.

Keywords and phrases. Closure operation, semiprime operation, Nakayama closure.

Received by the editors on January 11, 2012, and in revised form on May 6, 2013. 
semiprime and satisfies the additional assumption

$$
(0)^{c}=\bigcap_{n \geq 1}\left(I^{n}\right)^{c}
$$

for any $I$. The radical on rings of dimension at least one and the semiprime operations defined on discrete valuation rings which do not satisfy

$$
(0)^{c}=\bigcap_{n \geq 1}\left(I^{n}\right)^{c}
$$

for some $I$ are examples of semiprime operations which are not Nakayama. We are also able to exhibit some examples of closure operations, even in rings of dimension one, which are Nakayama but not semiprime. One of these closures is Mono(-), the monomial support of an ideal in a polynomial ring. We give further credence to the possibility that when $c$ is semiprime and satisfies $(0)^{c}=\cap_{n \geq 1}\left(I^{n}\right)^{c}$, then $c$ is Nakayama, by exhibiting this fact on the cuspidal cubic.

We now give a brief outline of the paper. In Section 2, we will discuss closure operations and some related operations: $c$-operations. We give several examples to highlight the various properties a closure operation may satisfy. Section 3 contains the results that we discussed in the previous paragraph.

2. Preliminaries. Let $R$ be a commutative ring and $\mathcal{I}$ a set of ideals of $R$ satisfying the property if $I \in \mathcal{I}$ and $I \subseteq J$, then $J \in \mathcal{I}$. There are many properties in the literature that a function $c: \mathcal{I} \rightarrow \mathcal{I}$ may satisfy. For example,

(C1) (The expansive property). $I \subseteq c(I)$ for all ideals $I \in \mathcal{I}$.

(C2) (The order preserving property). If $I \subseteq J$, then $c(I) \subseteq c(J)$.

(C3) (Idempotence). $c(I)=c(c(I))$.

For the rest of the paper, we will denote $c(I):=I^{c}$.

Definition 2.1. Let $R$ be a commutative ring and $\mathcal{I}$ a set of ideals of $R$ satisfying the property if $I \in \mathcal{I}$ and $I \subseteq J$, then $J \in \mathcal{I}$. If $c: \mathcal{I} \rightarrow \mathcal{I}$ satisfies (C1)-(C3) above, we call $c$ a closure operation on $\mathcal{I}$. If $\mathcal{I}$ is the set of all ideals in $R$, we call $c$ a closure operation on $R$. 
Ratliff and Rush modified these properties slightly and defined a function $c: \mathcal{I} \rightarrow \mathcal{I}$ to be a c-operator if (C1) holds and as long as $I \subseteq J \subseteq I^{c}$, then $I^{c}=J^{c}[8]$. This second weaker property implies the idempotence of $c$ but only gives the order preserving property for $c$-reductions of $I$, ideals sandwiched between $I$ and its "closure" $I^{c}$.

Further properties that a closure operation or a $c$-operator may satisfy are the following:

- If $\mathcal{I}$ is additionally multiplicatively closed, we say that $c$ satisfies the semiprime property if $I^{c} J^{c} \subseteq(I J)^{c}$. If $c$ is a closure operation, then we will call $c$ a semiprime operation.

- A semiprime operation $c$ is prime if $a I^{c}=(a I)^{c}$ for any regular element $a$. If $R$ is a domain, these operations are also called star operations. In a recent paper, [3], Epstein extended this notion to non-domains.

- If $(R, \mathfrak{m})$ is a local ring, then we say that a closure $c$ satisfies the Nakayama property if

$$
I \subseteq J \subseteq(I+\mathfrak{m} J)^{c} \text { then } I^{c}=J^{c} .
$$

Epstein called a closure operation with the Nakayama property a Nakayama closure in 2005 [1]. Prior to this, in 2002, Ratliff and Rush in [8] called a $c$-operation with the Nakayama property a $c^{*}$-operator. We have named this property after Nakayama because of its obvious relationship to Nakayama's lemma.

Certainly, if $\mathcal{M}$ is a set of all submodules of an $R$-module $M$, we can similarly consider functions $c: \mathcal{M} \rightarrow \mathcal{M}$ satisfying $(\mathrm{C} 1)-(\mathrm{C} 3)$ and the additional properties above. These are called closure operations on $M$. Although we don't concern ourselves with closure operations defined on $M$, this is discussed in papers of Epstein [2] and Kirby [6]. Also, for a nice overview on closure operations on rings and modules, please refer to Epstein's recent guide, [3]. We include some common examples below.

Our first example is the integral closure of an ideal, $I$. We say $r \in R$ is in the integral closure, $\bar{I}$ of $I$, if there exists a monic polynomial $p(x)=x^{n}+a_{1} x^{n-1}+\cdots+a_{n}$ with $a_{i} \in I^{i}$ such that $p(r)=0$. Note that the integral closure is always semiprime. If $R$ is a normal domain, 
then the integral closure is prime and, if $R$ is local ring, then $I \mapsto \bar{I}$ is Nakayama.

Let $R$ be a Noetherian ring of prime characteristic $p>0$. Denote positive powers of $p$ by $q$ and $R^{0}=R \backslash \cup P$ where $P$ ranges over the set of minimal primes of $R$. If $I=\left(x_{1}, \ldots, x_{n}\right)$, we define $I^{[q]}=\left(x_{1}^{q}, \ldots, x_{n}^{q}\right)$. We say $x \in I^{*}$, the tight closure of $I$, if there exists a $c \in R^{o}$ such that $c x^{q} \in I^{[q]}$ for all large $q$. The tight closure is also a semiprime operation. Tight closure of a principal ideal is equal to its integral closure in Noetherian domains with infinite residue field [5, Example 1.6.1]. This in conjunction with the fact that $(x I)^{*}: x=I^{*}[3$, Proposition 4.1.3] in a normal domain implies that tight closure is a prime operation for normal domains. Epstein has shown [1, Proposition 2.1] that if $R$ is a local ring with a weak test element, then $I \mapsto I^{*}$ is Nakayama.

The basically full closure of an $\mathfrak{m}$-primary ideal $I$ in a local ring $(R, \mathfrak{m})$ was introduced by Heinzer, Ratliff and Rush to be $(\mathfrak{m} I: \mathfrak{m})$. They showed that the basically full closure $I \mapsto I^{b f}$ on the set of $\mathfrak{m}$ primary ideals is semiprime [4, Theorem 4.2]. They also showed that $I \mapsto I^{b f}$ is a Nakayama closure [4, Lemma 5.4].

The Ratliff-Rush closure,

$$
\widetilde{I}=\bigcup_{i=1}^{\infty}\left(I^{n+1}: I^{n}\right)
$$

was introduced in 1977 by Ratliff and Rush in [9]. The Ratliff-Rush closure is a $c$-operator and not a closure operation since $\widetilde{I}$ is not order preserving (unless $I \subseteq \bar{I}[\mathbf{8}]$ ). However, $I \mapsto \widetilde{I}$ does satisfy the semiprime property $\widetilde{I} \widetilde{J} \subseteq \widetilde{I J}$. One can see this as follows: Suppose $a \in\left(I^{n+1}: I^{n}\right)$ and $b \in\left(J^{n+1}: J^{n}\right)$; then $a b \in\left((I J)^{n+1}:(I J)^{n}\right)$. Also, Ratliff and Rush show that $I \mapsto \widetilde{I}$ is a $c^{*}$-operator satisfying the Nakayama property in [8].

The radical, $\sqrt{I}=\left\{r \mid r^{n} \in I\right.$ for some $\left.n \in \mathbf{N}\right\}$, is a semiprime operation since for $r \in \sqrt{I}$ and $s \in \sqrt{J}$ we have $r^{n} \in I$ for some $n \in \mathbf{N}$ and $s^{m} \in J$ for some $m \in \mathbf{N}$ and $(r s)^{m+n}=r^{m} s^{n}\left(r^{n} s^{m}\right) \in I J$ implying that $r s \in \sqrt{I J}$. However, unless $R$ is zero-dimensional, the radical is not Nakayama. For example, Suppose $P \subsetneq \mathfrak{m}$ is a prime, then $P \subseteq \mathfrak{m} \subseteq \sqrt{\left(P+\mathfrak{m}^{2}\right)}=\mathfrak{m}$, but $P=\sqrt{P} \neq \mathfrak{m}$. However, as a $c$-operator, the radical is a $c^{*}$-operator. 
The author has classified the semiprime and prime operations on the set of ideals for Dedekind domains and the complete cuspidal cubic in [10]. For example, if $(R, P)$ is a discrete valuation ring, the nonzero ideals of $R$ can always be expressed in the form $P^{i}$ for some $i \geq 0$ and the ideals are totally ordered. The semiprime operations are either the identity operation or of the form $f_{n}$ or $g_{n}$ where

$$
f_{n}\left(P^{i}\right)=\left\{\begin{array}{l}
P^{i} \text { for } i<n \\
P^{n} \text { for } i \geq n
\end{array} \quad \text { and } f_{n}((0))=(0)\right.
$$

and

$$
g_{n}\left(P^{i}\right)=\left\{\begin{array}{l}
P^{i} \text { for } i<n \\
P^{n} \text { for } i \geq n
\end{array} \quad \text { and } g_{n}((0))=P^{n}\right.
$$

[10, Proposition 3.2]. The only prime operation is the identity [10, Proposition 3.4].

Recall that the Krull intersection theorem says that, if $(R, \mathfrak{m})$ is a Noetherian local domain and $I$ an ideal, then

$$
\bigcap_{i \geq 0} I^{i}=(0) .
$$

Definition 2.2. Let $R$ be a Noetherian ring. We will call a closure operation $c$ Krull if

$$
(0)^{c}=\left(\bigcap_{i \geq 0} I^{i}\right)^{c}=\bigcap_{i \geq 0}\left(I^{i}\right)^{c} .
$$

Not so surprisingly, we will see that $f_{n}$ are not Nakayama closures, whereas the $g_{n}$ are Nakayama closures.

Recall that, if $R$ is a domain with field of fractions $K$, a fractional ideal $I$ is a finitely generated $R$-submodule of $K$ satisfying the property $c I \subseteq R$ for some nonzero $c \in R$. If we extend $\mathcal{I}$ to include the fractional ideals of $R$, we define closure operations on the finitely generated $R$ submodules of $K$. We have noted in [11] that, if $R$ is a discrete valuation ring, then if $c$ is a semiprime operation on the set of fractional ideals of $R$, and $c$ must be the identity. 
3. Results. Nakayama closure operations on the set of ideals of a discrete valuation ring $(R, P)$ are semiprime as we will see in the next theorem. Closures which take $P^{i}$ to $P^{n}$ on a finite interval $n \leq i \leq m$ with $m \neq n$ such as the following example

$$
\left(P^{i}\right)^{c}=\left\{\begin{array}{l}
P \quad \text { for } 1 \leq i \leq 2 \\
P^{i} \quad \text { otherwise }
\end{array}\right.
$$

will never be Nakayama. Note that $P^{3} \subseteq P \subseteq\left(P^{3}+P^{2}\right)^{c}=P$ but $\left(P^{3}\right)^{c} \neq P^{c}$. Similarly, if $c=f_{n}$ is one the semiprime operation on a discrete valuation ring $(R, P)$ defined in [10, Proposition 3.2] then $c$ will not be Nakayama. Note that $(0) \subseteq P^{i}$ for all $i \geq 0$. If $c$ were Nakayama, then $(0) \subseteq P^{n} \subseteq\left(P^{n+1}\right)^{c}=P^{n}$, would imply that $(0)^{c}=\left(P^{n}\right)^{c}=P^{n}$. However, this is not the case. The previous example exhibits why we need to assume that our semiprime operation is also Krull in the following.

Theorem 3.1. Let $(R, P)$ be a discrete valuation ring. A closure operation $c$ on $R$ is a Nakayama closure if and only if it a semiprime operation which is Krull.

Proof. Suppose $c$ is a Nakayama closure. All the nonzero ideals in $R$ can be expressed of the form $P^{i}$ where $P$ is the maximal ideal and the ideals are totally ordered.

Suppose for some $j<k, P^{k} \subseteq P^{j} \subseteq\left(P^{k}+P^{j+1}\right)^{c}$. Since $c$ is Nakayama, then $\left(P^{k}\right)^{c}=\left(P^{j}\right)^{c}$. Because $\left(P^{j}\right)^{c} \supseteq P^{j}$, then $\left(P^{j}\right)^{c}=P^{i}$ for some $i \leq j$. For any $i \leq r \leq j,\left(P^{r}\right)^{c}=P^{i}$ since

$$
P^{i}=\left(P^{j}\right)^{c} \subseteq\left(P^{r}\right)^{c} \subseteq\left(P^{i}\right)^{c}=P^{i} .
$$

Similarly for $i \leq r \leq k,\left(P^{r}\right)^{c}=P^{i}$ since

$$
P^{i}=\left(P^{k}\right)^{c} \subseteq\left(P^{r}\right)^{c} \subseteq\left(P^{i}\right)^{c}=P^{i} .
$$

Note $\left.(0) \subseteq P^{k-1} \subseteq\left((0)+P P^{k-1}\right)\right)^{c}=\left(P^{k}\right)^{c}=P^{i}$. Now, by the Nakayama property, $(0)^{c}=\left(P^{k-1}\right)^{c}=P^{i}$. Combining this with the fact that $(0) \subseteq P^{r} \subseteq P^{k}$ for all $r>k$, we see that $\left(P^{r}\right)^{c}=P^{i}$ for all $r>k$. 
Suppose now that $P^{n} \subsetneq\left(P^{n}\right)^{c}=P^{m}$ for some $m<n<i$. Consider

$$
P^{i} \subseteq P^{m} \subseteq\left(P^{i}+P^{m+1}\right)^{c} \subseteq\left(P^{n}\right)^{c} .
$$

Again the Nakayama property would imply that $\left(P^{i}\right)^{c}=\left(P^{m}\right)^{c}=P^{m}$, contradicting that $\left(P^{i}\right)^{c}=P^{i}$. Thus, $\left(P^{n}\right)^{c}=P^{n}$ for all $n<i$.

Since

$$
(0)^{c}=\bigcap_{r \geq 0}\left(P^{r}\right)^{c}=P^{i},
$$

$c$ corresponds to the semiprime operation $g_{i}$ given in $[\mathbf{1 0}$, Proposition 3.4].

Now suppose that $I$ is semiprime and satisfies

$$
(0)^{c}=\bigcap_{i \geq 0}\left(P^{i}\right)^{c},
$$

then $c$ is determined by $g_{n}$ for some $n$ or is the identity map $e$. Then, clearly, $P^{i} \subseteq P^{j} \subseteq g_{n}\left(P^{i}+P^{j+1}\right)$. For, if $i \geq j \geq n$, then

$$
P^{i} \subseteq P^{j} \subseteq g_{n}\left(P^{i}+P^{j+1}\right)=P^{n}
$$

does imply that $g_{n}\left(P^{i}\right)=g_{n}\left(P^{j}\right)$, and if $i \geq n$,

$$
(0) \subseteq P^{i} \subseteq g_{n}\left(P^{i+1}\right)=P^{n}
$$

does imply $g_{n}(0)=g_{n}\left(P^{i}\right)$.

When the ring is not a discrete valuation ring, there are examples of Nakayama closures which are not semiprime. We illustrate a few below.

For the following two examples recall that the order of an ideal $I$ in a local ring $(R, \mathfrak{m})$ is

$$
o(I)=\left\{\begin{array}{l}
n \text { if } I \subseteq \mathfrak{m}^{n} \text { but } I \nsubseteq \mathfrak{m}^{n+1} \\
\infty \text { if } I \subset \mathfrak{m}^{n} \text { for all } n \in \mathbf{N} .
\end{array}\right.
$$

Definition 3.2. Let $(R, \mathfrak{m})$ be a local ring. The order operation on $R$ is

$$
\operatorname{ord}(I)=\left\{\begin{array}{l}
\mathfrak{m}^{o(I)} \text { if } o(I)<\infty \\
\bigcap_{n \geq 1} \mathfrak{m}^{n} \text { otherwise. }
\end{array}\right.
$$


Proposition 3.3. Let $(R, \mathfrak{m})$ be a local ring. Then the order operation is a closure operation on $R$.

Proof. We break the proof into the two cases:

1 ) when $o(I)$ is finite and 2) when $o(I)$ is infinite.

1) Suppose $o(I)=n$. Then $I \subseteq \mathfrak{m}^{n}=\operatorname{ord}(I)$. If $I \subseteq J$ then $o(I) \geq o(J)$ and ord $(I)=\mathfrak{m}^{o(I)} \subseteq \mathfrak{m}^{o(J)}=\operatorname{ord}(J)$. If ord $(I)=\mathfrak{m}^{n}$, then ord $\left(\mathfrak{m}^{n}\right)=\mathfrak{m}^{n}$ implies ord $(\operatorname{ord}(I))=\operatorname{ord}(I)$.

2) Now we assume that $o(I)=\infty$ implying $I \subseteq \mathfrak{m}^{n}$ for all $n \in \mathbf{N}$. Thus

$$
I \subseteq \bigcap_{n \geq 1} \mathfrak{m}^{n}=\operatorname{ord}(I)
$$

If $I \subseteq J$, then either $o(J)=m$ for some $m \in \mathbf{N}$ or $o(J)$ is infinite. In the first case

$$
\operatorname{ord}(I)=\bigcap_{n \geq 1} \mathfrak{m}^{n} \subseteq \mathfrak{m}^{m}=\operatorname{ord}(J)
$$

In the second case,

$$
\operatorname{ord}(I)=\bigcap_{n \geq 1} \mathfrak{m}^{n}=\operatorname{ord}(J),
$$

which again gives the containment ord $(I) \subseteq$ ord $(J)$. If

$$
\operatorname{ord}(I)=\bigcap_{n \geq 1} \mathfrak{m}^{n}
$$

then

$$
\bigcap_{n \geq 1} \mathfrak{m}^{n} \subseteq \mathfrak{m}^{n}
$$

for all $n \in \mathbf{N}$. Thus,

$$
\operatorname{ord}\left(\bigcap_{n \geq 1} \mathfrak{m}^{n}\right)=\bigcap_{n \geq 1} \mathfrak{m}^{n}=\operatorname{ord}(I) .
$$

We will use the following observations to justify that the following examples are Nakayama. 
Observation 3.4. In a local $\operatorname{ring}(R, \mathfrak{m})$, for ideals $I, J \subseteq R$,

$$
o(I+J)=\min \{o(I), o(J)\} .
$$

As a consequence, we obtain

$$
\operatorname{ord}(I+J)=\mathfrak{m}^{\min \{o(I), o(J)\}} .
$$

Similarly, for ideals $I_{1}, \ldots, I_{n} \subseteq R$,

$$
o\left(I_{1}+\cdots+I_{n}\right)=\min \left\{o\left(I_{1}\right), \ldots, o\left(I_{n}\right)\right\} .
$$

Proof. The elements of $I+J$ are of the form $x+y$ for some $x \in I$ and $y \in J$. First, suppose $o(I)=\infty=o(J)$. Then $I, J \subseteq \mathfrak{m}^{n}$ for all $n \in \mathbf{N}$. Now, for all $x \in I, y \in J$ and $n \in \mathbf{N}$ we have that $x \in I \subseteq \mathfrak{m}^{n}$, $y \in J \subseteq \mathfrak{m}^{n}$ and $x+y \in \mathfrak{m}^{n}$. Thus, $I+J \subseteq \mathfrak{m}^{n}$ for all $n \in \mathbf{N}$ and $o(I+J)=\infty=\min \{o(I), o(J)\}$. Now assume $o(I)=n$ is finite and $o(J)$ is infinite. Since $o(I)=n$, there exists an $x \in I \subseteq I+J$ such that $x \in \mathfrak{m}^{n} \backslash \mathfrak{m}^{n+1}$. Noting that

$$
x+\bigcap_{n \geq 1} \mathfrak{m}^{n} \subseteq \mathfrak{m}^{n} \quad \text { but } \quad x+\bigcap_{n \geq 1} \mathfrak{m}^{n} \nsubseteq \mathfrak{m}^{n+1},
$$

we will have $o(I+J)=n=\min \{o(I), o(J)\}$. The only case left to consider is if $o(I)=n$ and $o(J)=m$ are both finite. Let $x \in \mathfrak{m}^{n} \backslash \mathfrak{m}^{n+1}$ and $y \in \mathfrak{m}^{m} \backslash \mathfrak{m}^{m+1}$. Then $x, y \in I+J$, and clearly,

$$
o(I+J)=\min (n, m)=\min \{o(I), o(J)\} .
$$

By induction, we obtain

$$
o\left(I_{1}+\cdots+I_{n}\right)=\min \left\{o\left(I_{1}\right), \ldots, o\left(I_{n}\right)\right\} .
$$

Observation 3.5. In a local ring $(R, \mathfrak{m}), o(I J) \geq o(I)+o(J)$.

Proof. Suppose $o(I)=\infty$ or $o(J)=\infty$, then

$$
o(I J)=\infty=o(I)+o(J) .
$$

Now assume that $o(I)=n$ and $o(J)=m$ for $n, m \in \mathbf{N}$. Then, for some $x \in I$ and $y \in J, x \in \mathfrak{m}^{n} \backslash \mathfrak{m}^{n+1}$ and $y \in \mathfrak{m}^{m} \backslash \mathfrak{m}^{m+1}$. Now $x y \in \mathfrak{m}^{n+m}$, implying that $o(x y) \geq n+m$. Every element of $I J$ is of the form

$$
\sum_{i=1}^{n} x_{i} y_{i}
$$


From Observation 3.4, we know that

$$
o\left(\sum_{i=1}^{n} x_{i} y_{i}\right)=\min \left\{o\left(x_{1} y_{1}\right), \ldots o\left(x_{n} y_{n}\right)\right\} .
$$

Putting these facts together, we obtain $o(I J) \geq o(I)+o(J)$.

For the remainder of the paper we will assume that $k$ is a field.

Example 3.6. Take the ring $R=k[[x, y]] /(x y)$. Consider the order operation on $R$. Note that ord $\left(x+y^{2}\right)$ ord $\left(y+x^{2}\right)=\mathfrak{m}^{2}$ and since $\left(x+y^{2}\right)\left(y+x^{2}\right)=\left(x^{3}+y^{3}\right)$, then ord $\left(x^{3}+y^{3}\right)=\mathfrak{m}^{3}$. Clearly, $\mathfrak{m}^{2} \nsubseteq \mathfrak{m}^{3}$ implies the order operation is not semiprime. The order operation is Nakayama. Note if $I \subseteq J$ with $I \subseteq J \subseteq \operatorname{ord}(I+\mathfrak{m} J)$, then

$$
o(I) \geq o(J) \geq o(\operatorname{ord}(I+\mathfrak{m} J))=\min \{o(I), o(\mathfrak{m} J)\},
$$

where the last equality follows from Observation 3.4. If

$$
\min (o(I), o(\mathfrak{m} J))=o(I),
$$

then $o(I)=o(J)$ implies ord $(I)=\operatorname{ord}(J)$. If

$$
\min \{o(I), o(\mathfrak{m} J)\}=o(\mathfrak{m} J),
$$

then by Observation 3.5, we obtain $o(J) \geq o(J)+1$, which can only be the case if $o(J)=o(I)=\infty$ and, since $R$ is Noetherian, this means that $I=J=(0)$.

Example 3.7. Take the ring $k\left[\left[x^{2}, x^{3}\right]\right]$. Consider the order operation on $R$. Since $o\left(\left(x^{3}, x^{4}\right)\right)=1$, we see that $\left(\operatorname{ord}\left(x^{3}, x^{4}\right)\right)^{2}=\mathfrak{m}^{2}$, but

$$
o\left(\left(x^{3}, x^{4}\right)^{2}\right)=o\left(\left(x^{6}, x^{7}\right)\right)=o\left(\mathfrak{m}^{3}\right)=3,
$$

implies ord $\left(x^{3}, x^{4}\right)^{2}=\mathfrak{m}^{3}$. As in the previous example, $\mathfrak{m}^{2} \nsubseteq \mathfrak{m}^{3}$ again implies that the order operation is not semiprime. The order operation is Nakayama as in the previous example.

The order operation can be semiprime. Clearly, the order operation is the identity map on a discrete valuation ring $(R, P)$ which is semiprime. The order operation on any power series ring $k\left[\left[x_{1}, \ldots, x_{n}\right]\right]$ or localized polynomial ring $k\left[x_{1}, \ldots, x_{n}\right]_{\left(x_{1}, \ldots, x_{n}\right)}$ is semiprime since $o(I)=\min \{o(f) \mid f \in I\}$. Now, if $f \in I$ with $o(f)=o(I)$ 
and $g \in J$ with $o(g)=o(J)$, then $o(I J)=o(f g)=o(f)+o(g)$. Clearly, $o(f g) \geq o(f)+o(g)$ by Observation 3.5, but the other inequality is straightforward since both the power series ring the localized polynomial ring over a field have no zero divisors. Thus, ord $(I) \operatorname{ord}(J)=\operatorname{ord}(I J)$.

Before discussing our next example, we consider the monomial support of an ideal $I$, Mono $(I)$, defined in [7] on the ring $k\left[x_{1}, \ldots, x_{d}\right]$. Polini, Ulrich and Vitulli discuss Mono $(I)$ in terms of an algorithm for computing the core of an ideal. We will see that Mono is a closure operation.

Remark 3.8. Let $R=k\left[x_{1}, \ldots, x_{d}\right]$. A monomial is a polynomial of the form $x_{1}^{a_{1}} \cdots x_{d}^{a_{d}}$ with $a_{i} \in \mathbf{N}_{0}$ for $i=1, \ldots, d$. As shorthand, we will write $x^{\alpha}:=x_{1}^{a_{1}} \cdots x_{d}^{a_{d}}$ where $\alpha=\left(a_{1}, \ldots, a_{d}\right) \in \mathbf{N}_{0}^{d}$. Let $A$ be a finite subset of $\mathbf{N}_{0}^{d}$ and $0 \neq b_{\alpha} \in k$. Any polynomial $f \in R$ can be expressed in the form

$$
\sum_{\alpha \in A} b_{\alpha} x^{\alpha}
$$

The monomial support of $f$ is the ideal Mono $(f):=\left(x^{\alpha} \mid \alpha \in A\right)$. For any ideal $I \subseteq R, I=\left(f_{1}, \ldots, f_{n}\right)$. We define

$$
\operatorname{Mono}(I)=\sum_{i=1}^{n} \operatorname{Mono}\left(f_{i}\right) \text {. }
$$

This is the smallest monomial ideal containing $I$. If $I=(0)$, then Mono $(I)=(0)$. We will often denote a monomial by $m, m_{i}$ or $m_{i j}$ instead of $x^{\alpha}$ for ease of expressing the elements in $R$.

Proposition 3.9. Let $R=k\left[x_{1}, \ldots, x_{d}\right]$. Mono is a closure operation on the set of ideals of $R$.

Proof. Note that $I \subseteq \operatorname{Mono}(I)$ by definition.

Let $J=\left(f_{1}, \ldots f_{n}\right)$. Suppose

$$
f_{i}=\sum_{j=1}^{r_{i}} a_{j} m_{i j},
$$


where $0 \neq a_{j} \in k$ and $m_{i j}$ are monomials. Then

$$
\operatorname{Mono}(J)=\left(m_{i j} \mid 1 \leq i \leq n, 1 \leq j \leq r_{i}\right) .
$$

Suppose $g \in I \subseteq J$. Thus,

$$
g=g_{1} f_{1}+\cdots g_{n} f_{n}=\sum_{i=1}^{n} \sum_{j=1}^{r_{i}} g_{i} a_{j} m_{i j} .
$$

Since $g_{i}$ has order at least 0 , then the monomials in $g$ are contained in Mono $(J)$. This implies that if $I \subseteq J$, Mono $(I) \subseteq \operatorname{Mono}(J)$.

Since the smallest monomial ideal containing a monomial ideal is itself, then Mono $(\operatorname{Mono}(I))=\operatorname{Mono}(I)$.

The following lemmas illustrate some additional properties that Mono satisfies.

Lemma 3.10. Let $R=k\left[x_{1}, \ldots, x_{d}\right]$. For ideals $I, J \subseteq R$,

$$
\text { Mono }(I+J)=\operatorname{Mono}(I)+\operatorname{Mono}(J) \text {. }
$$

Proof. For monomial ideals $K$ and $L$, to show that $K \subseteq L$, it is enough to show that any monomial $m \in K$ is also a monomial in $L$. Let $m \in$ Mono $(I+J)$ be a monomial. Then $m$ is a monomial appearing in some polynomial $h \in I+J$. Since $h=f+g$ for some $f \in I$ and $g \in J$, then $m$ is either a monomial appearing in $f$ or $g$, thus

$$
m \in \operatorname{Mono}(I) \subseteq \operatorname{Mono}(I)+\operatorname{Mono}(J)
$$

or

$$
m \in \operatorname{Mono}(J) \subseteq \operatorname{Mono}(I)+\operatorname{Mono}(J) .
$$

Note that Mono $(I) \subseteq \operatorname{Mono}(I+J)$ and Mono $(J) \subseteq \operatorname{Mono}(I+J)$ since Mono is a closure operation. Thus

$$
\operatorname{Mono}(I)+\operatorname{Mono}(J) \subseteq \operatorname{Mono}(I+J) .
$$

Lemma 3.11. Let $R=k\left[x_{1}, \ldots, x_{d}\right]$ and $I \subseteq R$ an ideal. For any monomial $m$,

$$
\operatorname{Mono}(m I)=m \operatorname{Mono}(I) \text {. }
$$


Proof. For any $f \in I, f=a_{1} m_{1}+a_{2} m_{2}+\cdots+a_{n} m_{n}$ for $0 \neq a_{i} \in k$ and $m_{i}$ a monomial. Then $m f=a_{1} m m_{1}+a_{2} m m_{2}+\cdots+a_{n} m m_{n}$ is a typical element in $m I$. Thus, Mono $(m f)=\left(m m_{1}, \ldots m m_{n}\right)=$ $m$ Mono $(f)$. Let $I=\left(f_{1}, \ldots, f_{r}\right)$. By definition of Mono, we see

$$
\operatorname{Mono}(m I)=\sum_{i=1}^{r} \operatorname{Mono}\left(m f_{i}\right)=m \sum_{i=1}^{r} \operatorname{Mono}\left(f_{i}\right)=m \operatorname{Mono}(I) \text {. }
$$

Lemma 3.12. Let $R=k\left[x_{1}, \ldots, x_{d}\right]$ with $\mathfrak{m}=\left(x_{1}, \ldots, x_{d}\right)$ and $I \subseteq R$ be an ideal. Then Mono $(\mathfrak{m} I)=\mathfrak{m M o n o}(I)$. Thus,

Proof. By Lemma $3.11 x_{j} \operatorname{Mono}(I)=\operatorname{Mono}\left(x_{j} I\right)$ for all $j=1, \ldots d$.

$$
\begin{aligned}
\mathfrak{m} \operatorname{Mono}(I) & =x_{1} \operatorname{Mono}(I)+x_{2} \operatorname{Mono}(I)+\cdots+x_{d} \operatorname{Mono}(I) \\
& =\operatorname{Mono}\left(x_{1} I\right)+\operatorname{Mono}\left(x_{2} I\right)+\cdots+\operatorname{Mono}\left(x_{d} I\right) \\
& =\operatorname{Mono}\left(x_{1} I+x_{2} I+\cdots+x_{d} I\right) \\
& =\operatorname{Mono}(\mathfrak{m} I)
\end{aligned}
$$

where the second to last equality follows from Lemma 3.10.

Example 3.13. Take the ring $k[x, y]_{(x, y)}$ with closure Mono. Consider $I=(x+y)$ and $J=(x-y)$. Mono $(I)=\mathfrak{m}=\operatorname{Mono}(J)$, but

$$
\operatorname{Mono}(I J)=\operatorname{Mono}\left(x^{2}-y^{2}\right)=\left(x^{2}, y^{2}\right) \nsupseteq \mathfrak{m}^{2}=\operatorname{Mono}(I) \operatorname{Mono}(J) .
$$

Hence, Mono is not semiprime.

The closure Mono is Nakayama, since if $I \subseteq J \subseteq \operatorname{Mono}(I+\mathfrak{m} J)$, then clearly Mono $(I) \subseteq \operatorname{Mono}(J) \subseteq \operatorname{Mono}(I+\mathfrak{m} J)$. Suppose Mono $(J)$ is minimally generated by $m_{1}, \ldots, m_{n}$. Then

$$
m_{i} \notin \mathfrak{m} \operatorname{Mono}(J)=\operatorname{Mono}(\mathfrak{m} J)
$$

by Lemma 3.12. Lemma 3.10 implies that

$$
\operatorname{Mono}(I+\mathfrak{m} J)=\operatorname{Mono}(I)+\operatorname{Mono}(\mathfrak{m} J) .
$$

Hence, Mono $(J) \subseteq$ Mono $(I)+\operatorname{Mono}(\mathfrak{m} J)$ imply that the monomials in Mono $(J)$ must be contained in Mono $(I)$ implying Mono $(I)=$ $\operatorname{Mono}(J)$. 
The author believes that all semiprime operations defined on onedimensional domains which are Krull will be Nakayama. Some evidence of this can be seen in the following.

Proposition 3.14. Let $R=k\left[\left[x^{2}, x^{3}\right]\right]$ and $I \rightarrow I^{c}$ a semiprime operation which is Krull, then $I \rightarrow I^{c}$ is Nakayama.

Proof. The author, in [10, Theorem 4.8, Theorem 4.9], classified all the semiprime operations on $R$. All the ideals of $k\left[\left[x^{2}, x^{3}\right]\right]$ are minimally generated by at most two elements. We denote the nonzero nonunit ideals

$$
M_{i}=\left(x^{i}, x^{i+1}\right) \quad \text { and } \quad P_{i, a}=\left(x^{i}+a x^{i+1}\right)
$$

for $i \geq 2$ and $a \in k$. Note that the maximal ideal is $M_{2}$. The semiprime operations which are Krull are the following, where $S$ and $T$ are subsets of the field $k$ :

(1) $f_{i, S, T}^{\text {int }}(I)=\left\{\begin{array}{c}I \text { if } I \supseteq P_{i, a}, a \notin S \text { or } I \supseteq P_{i+1, b}, b \notin T \\ \bar{I} \text { if } I \subseteq M_{i+2}, \quad I=P_{i, a}, a \in S \\ \text { or } I=P_{i+1, b}, b \in T\end{array}\right.$

(2) $g_{m, a}^{f}(I)=\left\{\begin{array}{l}I \text { for } I \supseteq P_{m, a} \\ M_{m-1} \text { for } I=P_{m-1, b}, \text { for all } b \in k \\ M_{m} \text { for } I=P_{m, b}, \quad b \neq a, I=P_{m+1, d}, \\ \quad \text { for all } d \in k \text { or } I=M_{m+1} \\ P_{m, a} \text { for } I \subseteq P_{m, a}\end{array}\right.$ for $m \geq 2$ and $a \in k$,

(3) $g_{n, S, T, m}^{f}(I)=\left\{\begin{array}{l}I \text { for } I \supseteq P_{n, a}, a \notin S \text { or } I \supseteq P_{n+1, b}, b \notin T \\ \bar{I} \text { for } P_{m-1, d} \subseteq I \subseteq J, J=P_{n, a}, a \in S \\ \quad \text { or } J=P_{n+1, b}, b \in T \\ M_{m} \text { for } I \subseteq M_{m}\end{array}\right.$ for $m-1 \geq n \geq 2, S \neq \emptyset$ and, if $m=n+1, T=k$,

(4) $g_{n, S, T, m \prime}^{f}(I)=\left\{\begin{array}{l}I \text { for } I \supseteq P_{n, a}, \quad a \notin S \text { or } I \supseteq P_{n+1, b}, b \notin T \\ \bar{I} \text { for } M_{m-2} \subseteq I \subseteq J, J=P_{n, a}, a \in S \\ \quad \text { or } J=P_{n+1, b}, b \in T \\ M_{m-2} \text { for } I=P_{m-1, d}, M_{m-1} \\ M_{m} \text { for } I \subseteq M_{m}\end{array}\right.$ for $m-2 \geq n \geq 2, S \neq \emptyset$.

It is straightforward to check that all of these semiprime operations 
are also Nakayama closures. For example, for $a \in S$,

$$
P_{i, a} \subseteq M_{i} \subseteq f_{i, S, T}^{\mathrm{int}}\left(P_{i, a}+M_{i} M_{2}\right)=f_{i, S, T}^{\mathrm{int}}\left(P_{i, a}\right)=M_{i}
$$

and $f_{i, S, T}^{\mathrm{int}}\left(P_{i, a}\right)=f_{i, S, T}^{\mathrm{int}}\left(M_{i}\right)$.

Note that, if $c$ is a semiprime operation on a one-dimensional domain satisfying $\mathfrak{m}^{c}=R$, then since all $\mathfrak{m}$-primary ideals $I$ satisfy $\mathfrak{m}^{n} \subseteq I$ for some $n \geq 1$, we can conclude that $R=\left(\mathfrak{m}^{c}\right)^{n} \subseteq I^{c}$ since ${ }^{c}$ is semiprime. Thus, $I^{c}=R$ for all $\mathfrak{m}$-primary ideals. Now since $c$ is Krull, then $(0)^{c}=R$ and all ideals satisfy $J \subseteq I \subseteq(J+\mathfrak{m} I)^{c}$ implies $J^{c}=I^{c}=R$.

If $c$ is a semiprime operation satisfying $\mathfrak{m}^{c}=\mathfrak{m}$, then since $c$ is semiprime,

$$
\mathfrak{m} I^{c}=\mathfrak{m}^{c} I^{c} \subseteq(\mathfrak{m} I)^{c} .
$$

Suppose $J \subseteq I$. Then $J^{c} \subseteq I^{c}$ since semiprime operations are order preserving. If $I$ satisfies $I^{c} \subseteq(J+\mathfrak{m} I)^{c}$, then we do not necessarily have that $I^{c} \subseteq J^{c}+\mathfrak{m} I^{c}$. If we knew this, then $I^{c}=J^{c}+\mathfrak{m} I^{c}$. Now, by Nakayama's lemma, $I^{c}=J^{c}$ and $c$ is a Nakayama closure. In the above theorem, we see that all the semiprime operations defined on $k\left[\left[x^{2}, x^{3}\right]\right]$ actually do satisfy the stronger condition $I^{c} \subseteq J^{c}+\mathfrak{m} I^{c}$ where we can apply Nakayama's lemma. It may be the case that all semiprime operations which are Krull will be Nakayama if $R$ is a one-dimensional domain, but at this time we have no way of generalizing the above proof.

Acknowledgments. The author would like to thank Jessie Elliot for asking a question which led to this note. Also, she extends much thanks to the referee for his/her suggestions on improving the exposition of the paper.

\section{REFERENCES}

1. N. Epstein, A tight closure analogue of analytic spread, Math. Proc. Camb. Phil. Soc. 139 (2005), 371-383.

2. Reductions and special parts of closures, J. Algebra 323 (2010), 2209-2225.

3. Comm. Alg. II, Walter de Gruyter, Berlin, 2012.

4. W. Heinzer, L. Ratliff and D. Rush, Basically full ideals in local rings, J. Alg. 250 (2002), 371-396. 
5. C. Huneke, Tight closure and its applications, CBMS Lect. Notes Math. 88, American Mathematical Society, Providence 1996.

6. D. Kirby, Closure operations on ideals and submodules, J. Lond. Math. Soc. 44 (1969), 283-291.

7. C. Polini, B. Ulrich and M. Vitulli, The core of zero-dimensional monomial ideals, Adv. Math. 211 (2007), 72-93.

8. L. Ratliff and D. Rush, Asymptotic primes of delta closures of ideals, Comm. Alg. 30 (2002), 1513-1531.

9. Notes on ideal covers and associated primes, Pacific J. Math. 73 (1977), 169-191.

10. J. Vassilev, Structure on the set of closure operations of a commutative ring, J. Algebra 321 (2009), 2737-2753.

11. A look at the prime and semiprime operations of onedimensional domains, Houst. J. Math. 38 (2012), 1-15.

University of New Mexico, Albuquerque, NM

Email address: jvassil@math.unm.edu 